2

3

4

5

6

7

8

9

10

11

12

\title{
Photochemical processing of inorganic and organic species in the Canadian high Arctic aerosols: Impact of ammonium cation, transition metals, and dicarboxylic acids before and after polar sunrise at Alert
}

Dharmendra Kumar Singh ${ }^{*}$, Kimitaka Kawamura ${ }^{*}$,c , Pingqing Fu ${ }^{\text {d }}$, Hideki Kasukabe ${ }^{c}$, Ayako Yanase $^{c}$, and Leonard A. Barrie e,f,g

aFrench National Centre for Scientific Research (CNRS)/IRCELyon, Villeurbanne-69100, France

${ }^{\mathbf{b}}$ Chubu Institute for Advanced Studies, Chubu University, Kasugai 487-8501, Japan 'Department of Chemistry, Tokyo Metropolitan University, Hachioji, Tokyo 192-0397, Japan dThe Institute of Surface-Earth System Science, Tianjin University, Tianjin, 300072, China eAtmosphere and Ocean Sciences Department, McGill University, Montreal QC, Canada fBolin Centre Research, Stockholm University, Stockholm SE-106 91, Sweden g Environment and Climate Change Canada, Toronto, Ontario M3H 5T4, Canada

*Corresponding author: kkawamura@,isc.chubu.ac.jp Tel: +81-568-51-9330, Fax: +81-568-51-473 dharmendraks841@gmail.com

\section{Contents of this file}

Table S1 to S8

Figure S1 to S5 
36 Table S1. Comparison of new findings of dataset (Not a single finding can be matched from our

\section{previously published articles) with published outcomes}

\section{New findings (Present study)}

1. Total dicarboxylic acid (TDCA) was contributed $89 \%$ of the total identified organic species followed by total oxoacids (9\%) and $\alpha$-dicarbonyls (2\%).

2. One of the Fenton reagents, Fe, is highly correlated with dicarboxylic acids before polar sunrise (in dark winter) and another $\mathrm{Cu}$ is highly correlated after polar sunrise.

3. After polar sunrise, dicarboxylic acids, oxoacids, and $\alpha$-dicarbonyls are substantially correlated with $\mathrm{NH}_{4}^{+}$which suggests the formation of ammonium salts in the Arctic atmosphere due to the photochemical formation of organic acids and emissions of $\mathrm{NH}_{3}$ from the ocean/land.

4. Strong correlations (upto $R^{2}=0.86, p<0.01$ ) of transition metals (Fe, Cu and Mn) with WSOC after polar sunrise and good correlation $\left(R^{2}=0.73 ; p<0.05\right)$ of oxalic acid with $\mathrm{Fe}$ in dark winter (before polar sunrise) hints the plausibility of the Fenton chemistry.

5. WSOC and diacids are highly correlated $\left(R^{2}=0.94, p>0.01\right)$ and (upto $\left.22=0.80 ; p<0.05\right)$ with $\mathrm{NH}_{4}^{+}$after polar sunrise, indicating the formation of possible organic salts.

6. $K^{+}$is highly correlated $\left(R^{2}=0.96 ; p=0.03\right)$ with levoglucosan, suggesting that biomass burning products transported from midlatitudes to the Arctic before polar sunrise.

7. $C a$ is significantly correlated $\left(R^{2}=0.99 ; p<0.01\right)$ with oxalic acid before polar sunrise signifies the possible formation of calcium oxalate in the winter dark.

8. Manganese $(\mathrm{Mn})$ is the most significant predictor $\left(R^{2}=0.86 ; p<0.001\right)$ for the formation of WSOC followed by Cu and $\mathrm{Fe}$ at the polar sunrise in multiple linear regression model.

9. First time, the highest (89\%) percentage ratios of WSOC/OC (70\% on average) were observed in mid-March and mid-April.

Published outcomes (Kawamura et al. 2010) ${ }^{19}$

- Water-soluble dicarboxylic acids (C2-C12), ketoearboxylic acids (C2-C6, C9), and a-dicarbonyls (glyoxal and methylglyoxal) were determined in the Arctic aerosols collected in winter to early summer, as well as aerosol total carbon (TC) and water-soluble organic carbon (WSOC).

- Concentrations of TC and WSOC gradually decreased from late February to early June with a peak in spring, indicating a photochemical formation of water-soluble organic aerosols at a polar sunrise.

- Kawamura et al (JGR, 2010). ${ }^{19}$ found that total (C2-C11) diacids (7-84 $\left.\mathrm{ng} \mathrm{m}{ }^{-3}\right)$ increased at polar sunrise by a factor of 4 and then decreased toward summer. Their contributions to TC (average 4.0\%) peaked in early April and mid-May.

- The contribution of total diacids to WSOC was on average $7.1 \%$. It gradually increased from February (5\%) to a maximum in April (12.7\%) with a second peak in mid-May (10.4\%).

- Although oxalic acid (C2) is the dominant diacid until April, its predominance was replaced by succinic acid (C4) after polar sunrise. This may indicate that photochemical production of $\mathrm{C} 2$ was overwhelmed by its degradation when solar radiation was intensified and the atmospheric transport of its precursors from midlatitudes to the Arctic was ended in May.

- Interestingly, the contributions of azelaic (C9) and w-oxo butanoic acids to WSOC increased in early summer possibly due to an enhanced emission of biogenic unsaturated fatty acids from the ocean followed by photochemical oxidation in the atmosphere. An enhanced contribution of diacids to TC and WSOC at polar sunrise may significantly alter the hygroscopic properties of organic aerosols in the Arctic. 
40 Table S2 Average temperature, concentrations of aerosol mass (TSPM) and major inorganic 41 and carbonaceous species measured in the Arctic aerosol

42

\begin{tabular}{lc}
\hline Compounds & Mean \\
\hline Ambient temperature $\left({ }^{\circ} \mathrm{C}\right)$ & -19.6 \\
Aerosol mass $\left(\mathrm{ng} \mathrm{m}^{-3}\right)$ & 5250 \\
$\mathrm{TC}$ & 359 \\
$\mathrm{TN}$ & 78.9 \\
$\mathrm{TC} /$ Aerosol mass \% & $6.27 \%$ \\
$\mathrm{TN} /$ Aerosol mass \% & $1.41 \%$ \\
$\mathrm{C} / \mathrm{N}$ weight ratios & 4.61
\end{tabular}

Major Inorganic metals

Aluminium

Magnisium

Calcium

Iron

Minor Inorganic metals

Copper

Zinc

Vanadium

Lead

Nickle

Magnese

Major Inorganic ions

Chloride

Nitrate

Sulphate

Non-sea-salt-sulphate

Sodium

Ammonium

Potasium ion

Magnisium ion

Calcium ion

Carbonaceous species

Elemental carbon

Organic carbon

Water-soluble organic carbon (ng $\mathrm{C} \mathrm{m}^{-3}$ )

WSOC/TC ratios, \%

$\mathrm{WSOC} /$ aerosol mass ratios, $\%$
Symbol/Abbreviation $\quad$ Mean \pm Standard deviation $\left(\mathrm{ng} \mathrm{m}^{-3}\right)$

$\begin{array}{ll}\mathrm{Al} & 48.85 \pm 29.44 \\ \mathrm{Mg} & 48.01 \pm 49.62 \\ \mathrm{Ca} & 46.02 \pm 29.65 \\ \mathrm{Fe} & 26.95 \pm 13.62\end{array}$

$\mathrm{Cu}$

$0.86 \pm 0.57$

$\mathrm{Zn}$

$1.43 \pm 2.17$

$\mathrm{V}$

$0.27 \pm 0.21$

$\mathrm{Pb}$

$1.02 \pm 0.83$

$\mathrm{Ni}$

$0.14 \pm 0.11$

$\mathrm{Mn}$

$0.58 \pm 0.35$

$\begin{array}{cc}\mathrm{Cl}^{-} & 95.51 \pm 178.17 \\ \mathrm{NO}_{3}{ }^{-} & 26.79 \pm 25.95 \\ \mathrm{SO}_{4}{ }^{2-} & 1120.35 \pm 634.27 \\ \mathrm{nssSO}_{4}{ }^{2-} & 1088.28 \pm 621.22 \\ \mathrm{Na}^{+} & 127.82 \pm 120.55 \\ \mathrm{NH}_{4}^{+} & 112.95 \pm 50.21 \\ \mathrm{~K}^{+} & 11.39 \pm 10.19 \\ \mathrm{Mg}^{2+} & 32.96 \pm 36.16 \\ \mathrm{Ca}^{2+} & 38.90 \pm 25.39\end{array}$

EC

$62.32 \pm 46.46$

$\mathrm{OC}$

$253.84 \pm 91.38$

WSOC

$186 \pm 86.50$

$55.7 \%$

$3.41 \%$ 
Table S3. Average concentrations $\left(\mathrm{ng} \mathrm{m}^{-3}\right)$ of series of major and minor saturated diacids, $\omega$ oxocarboxylic acids and ketoacids

\begin{tabular}{|c|c|c|c|}
\hline Major saturated dicarboxylic acid & Chemical formula & Abbreviations & Mean \\
\hline Oxalic & HOOC-COOH & $\mathrm{C}_{2}$ & 16.84 \\
\hline Malonic & $\mathrm{HOOC}-\mathrm{CH}_{2}-\mathrm{COOH}$ & $\mathrm{C}_{3}$ & 4.85 \\
\hline Succinic & $\mathrm{HOOC}-\left(\mathrm{CH}_{2}\right)_{2}-\mathrm{COOH}$ & $\mathrm{C}_{4}$ & 5.68 \\
\hline Glutaric & $\mathrm{HOOC}-\left(\mathrm{CH}_{2}\right)_{3}-\mathrm{COOH}$ & $\mathrm{C}_{5}$ & 2.03 \\
\hline Adipic & $\mathrm{HOOC}-\left(\mathrm{CH}_{2}\right)_{4}-\mathrm{COOH}$ & $\mathrm{C}_{6}$ & 1.42 \\
\hline \multicolumn{4}{|l|}{ Minor saturated dicarboxylic acid } \\
\hline Pimelic & $\mathrm{HOOC}-\left(\mathrm{CH}_{2}\right)_{5}-\mathrm{COOH}$ & $\mathrm{C}_{7}$ & 0.11 \\
\hline Suberic & $\mathrm{HOOC}-\left(\mathrm{CH}_{2}\right)_{6}-\mathrm{COOH}$ & $\mathrm{C}_{8}$ & 0.10 \\
\hline Azelaic & $\mathrm{HOOC}-\left(\mathrm{CH}_{2}\right)_{7}-\mathrm{COOH}$ & $\mathrm{C}_{9}$ & 0.10 \\
\hline Decanedioic & $\mathrm{HOOC}-\left(\mathrm{CH}_{2}\right)_{8}-\mathrm{COOH}$ & $\mathrm{C}_{10}$ & 0.029 \\
\hline Undecanedioic & $\mathrm{HOOC}-\left(\mathrm{CH}_{2}\right)_{9}-\mathrm{COOH}$ & $\mathrm{C}_{11}$ & 0.063 \\
\hline \multicolumn{4}{|l|}{ Unsaturated aliphatic diacids } \\
\hline Maleic & $\mathrm{HOOC}-\mathrm{CH}=\mathrm{CH}-\mathrm{COOH}$ & $\mathrm{M}$ & 0.23 \\
\hline Methylmaleic & $\mathrm{CH}_{3} \mathrm{OOC}-\mathrm{CH}=\mathrm{CH}-\mathrm{COOH}$ & $\mathrm{mM}$ & 0.11 \\
\hline Fumaric & $\mathrm{HOOC}-\mathrm{CH}=\mathrm{CH}-\mathrm{COOH}$ & $\mathrm{F}$ & 0.17 \\
\hline \multicolumn{4}{|l|}{ Unsaturated aromatic diacids } \\
\hline Phthalic & $\mathrm{HOOC}-\left(\mathrm{C}_{6} \mathrm{H}_{4}\right)-\mathrm{COOH}$ & $\mathrm{Ph}$ & 1.59 \\
\hline \multicolumn{4}{|l|}{$\omega$-Oxocarboxylic acids } \\
\hline Glyoxylic & $\mathrm{OHC}-\mathrm{COOH}$ & $\omega \mathrm{C} 2$ & 2.45 \\
\hline 3-oxopropanoic & $\mathrm{OHC}-\mathrm{CH}_{2}-\mathrm{COOH}$ & $\omega \mathrm{C} 3$ & 0.11 \\
\hline 4-oxobutanoic & $\mathrm{OHC}-\left(\mathrm{CH}_{2}\right)_{2}-\mathrm{COOH}$ & $\omega \mathrm{C} 4$ & 0.57 \\
\hline 6-oxopentanoic & $\mathrm{OHC}-\left(\mathrm{CH}_{2}\right)_{3}-\mathrm{COOH}$ & $\omega \mathrm{C5}$ & 0.069 \\
\hline 7-oxohexanoic & $\mathrm{OHC}-\left(\mathrm{CH}_{2}\right)_{4}-\mathrm{COOH}$ & $\omega \mathrm{C6}$ & 0.10 \\
\hline 8-oxononanoic & $\mathrm{OHC}-\left(\mathrm{CH}_{2}\right)_{7}-\mathrm{COOH}$ & $\omega \mathrm{C} 9$ & 0.03 \\
\hline \multicolumn{4}{|l|}{ Ketoacid } \\
\hline Pyruvic acid & $\mathrm{CH}_{3}-\mathrm{C}(\mathrm{O})-\mathrm{COOH}$ & Pyr & 0.29 \\
\hline \multicolumn{4}{|l|}{$\alpha$-Dicarbonyls } \\
\hline Glyoxal & $\mathrm{OHC}-\mathrm{CHO}$ & Gly & 0.55 \\
\hline Methylglyoxal & $\mathrm{CH}_{3}-\mathrm{C}(\mathrm{O})-\mathrm{CHO}$ & MGly & 0.32 \\
\hline
\end{tabular}


53 Table S4. Comparision of average concentrations ${ }^{\mathrm{a}}$ of diacids and related compounds measured 54 in the Arctic region with the different sites of South Asia including midlatitude (China).

\begin{tabular}{|c|c|c|c|c|c|c|}
\hline $\begin{array}{l}\text { Diacids and related } \\
\text { compounds }\end{array}$ & Arctic (Present study) ${ }^{a}$ & $\begin{array}{l}\text { South India } \\
\text { (Chennai) }^{\mathrm{b}}\end{array}$ & $\begin{array}{l}\text { Hong } \\
\text { Kong }^{\mathrm{c}}\end{array}$ & China $^{d}$ & $\begin{array}{c}\text { Japan } \\
\text { (Sapporo) }^{\mathrm{e}}\end{array}$ & Himalaya $^{f}$ \\
\hline \multicolumn{7}{|l|}{ Saturated diacids } \\
\hline Oxalic & 16.84 & 241 & 268 & 513 & 192 & 138 \\
\hline Malonic & 4.85 & 37.2 & 47.6 & 50.9 & 77 & 15.4 \\
\hline Succinic & 5.68 & 27.7 & 33.0 & 71.8 & 58 & 28.6 \\
\hline Glutaric & 2.03 & 11.4 & 6.95 & 30.0 & 13 & 4.86 \\
\hline Adipic & 1.42 & 9.4 & 12.7 & 23.5 & 5.1 & 2.34 \\
\hline Pimelic & 0.11 & 9.0 & 1.40 & 6.34 & 2.6 & 1.46 \\
\hline Suberic & 0.10 & 15.5 & 2.56 & 9.06 & 1.1 & 0.79 \\
\hline Azelaic & 0.10 & 32.4 & 9.06 & 31.6 & 6.5 & 2.24 \\
\hline Sebacic & 0.029 & 4.6 & 1.47 & 2.49 & 1.3 & 0.73 \\
\hline Undecanoic & 0.06 & 4.2 & 3.27 & 5.18 & 1.0 & 0.40 \\
\hline \multicolumn{7}{|l|}{ Unsaturated diacids } \\
\hline Maleic & 0.23 & 2.3 & 12.4 & 5.04 & 4.1 & 5.71 \\
\hline Fumaric & 0.17 & 1.3 & 3.38 & 2.47 & 2.1 & 1.20 \\
\hline Phthalic & 1.59 & 21.5 & 89.9 & 102 & 17 & 16.3 \\
\hline Total diacids & 33.20 & 502.9 & 526 & 892 & 406 & 235 \\
\hline \multicolumn{7}{|l|}{$\omega$-Oxocarboxylic acid } \\
\hline Glyoxylic, $\omega \mathrm{C}_{2}$ & 2.46 & 17.3 & 18.5 & 23.9 & 22 & 6.27 \\
\hline \multicolumn{7}{|l|}{$\alpha$-Dicarbonyls } \\
\hline Glyoxal & 0.55 & 2.9 & 0.19 & 2.72 & 4.6 & 0.84 \\
\hline Methylglyoxal & 0.32 & 4.2 & 10.0 & 2.47 & 5.1 & 0.98 \\
\hline Total $\alpha$-dicarbonyls & 0.87 & 7.1 & 10.2 & 5.19 & 9.7 & 1.82 \\
\hline
\end{tabular}

${ }^{\mathrm{a}}$ Concentrations are in $\mathrm{ng} \mathrm{m}^{-3} .{ }^{\mathrm{b}} \mathrm{PM}_{10}$ samples collected in summer [Pavuluri et al. 2010]. ${ }^{\mathrm{C}} \mathrm{PM}_{2.5}$ samples collected in summer [Ho et al. 2006]. ${ }^{\mathrm{d}} \mathrm{PM}_{2.5}$ samples collected in summer [Ho et al. 2007]. ${ }^{\mathrm{e}} \mathrm{TSP}$ samples collected in spring and summer [Aggarwal and Kawamura 2008]. ${ }^{\mathrm{f}}$ TSP samples collected in summer [Cong et al. 2015] 


\begin{tabular}{|c|c|c|c|c|c|c|}
\hline \multirow[b]{2}{*}{ DCA vs $\mathrm{NH}_{4}^{+}$} & \multicolumn{3}{|l|}{ Before Polar Sunrise } & \multicolumn{3}{|l|}{ After Polar Sunrise } \\
\hline & Regression equation & $\mathbf{R}^{2}$ & P-value & Regression equation & $\mathbf{R}^{2}$ & P-value \\
\hline $\mathrm{C}_{2} \mathrm{vs} \mathrm{NH}_{4}^{+}$ & $y=0.1007 x+5.884$ & 0.52 & $\mathrm{p}=0.36$ & $y=0.1544 x-0.4632$ & 0.58 & $\mathrm{p}=0.05$ \\
\hline $\mathrm{C}_{3} \mathrm{vs} \mathrm{NH}_{4}^{+}$ & $y=0.0622 x-3.7031$ & 0.70 & $\mathrm{p}<0.05$ & $y=0.045 x+0.3602$ & 0.80 & $\mathrm{p}<0.05$ \\
\hline $\mathrm{C}_{4} \mathrm{Vs} \mathrm{NH}_{4}^{+}$ & $y=0.0393 x-1.3681$ & 0.62 & $\mathrm{p}<0.05$ & $y=0.0447 x+1.8627$ & 0.49 & $\mathrm{p}>0.05$ \\
\hline $\mathrm{C}_{5} \mathrm{vs} \mathrm{NH}_{4}^{+}$ & $y=0.0162 x-0.335$ & 0.58 & $\mathrm{p}>0.05$ & $y=0.0271 x-0.7197$ & 0.59 & $\mathrm{p}>0.05$ \\
\hline $\mathrm{C}_{6} \mathrm{Vs} \mathrm{NH}_{4}^{+}$ & $y=0.0123 x-0.0045$ & 0.44 & $\mathrm{p}>0.05$ & $y=0.012 x+0.0914$ & 0.57 & $\mathrm{p}<0.05$ \\
\hline $\mathrm{C}_{7} \mathrm{vs} \mathrm{NH}_{4}^{+}$ & $y=0.0005 x+0.0982$ & 0.07 & $\mathrm{p}=0.66$ & $y=0.0015 x-0.0649$ & 0.40 & $\mathrm{p}=0.03$ \\
\hline $\mathrm{C}_{8} \mathrm{vs} \mathrm{NH}_{4}^{+}$ & $y=0.0008 x+0.0348$ & 0.47 & $\mathrm{p}>0.05$ & $y=0.0009 x-0.0039$ & 0.40 & $\mathrm{p}<0.05$ \\
\hline $\mathrm{C}_{9} \mathrm{vs} \mathrm{NH}_{4}^{+}$ & $y=0.0003 x+0.0992$ & 0.094 & $p=0.61$ & $y=0.0003 x+0.0992$ & 0.095 & $\mathrm{p}=0.17$ \\
\hline $\mathrm{C}_{10} \mathrm{vs} \mathrm{NH}_{4}^{+}$ & $y=7 E-06 x+0.004$ & 0.0005 & $\mathrm{p}=0.97$ & $y=0.0005 x-0.0088$ & 0.38 & $\mathrm{p}=0.06$ \\
\hline $\mathrm{C}_{11} \mathrm{vs} \mathrm{NH}_{4}^{+}$ & $y=0.0009 x-0.0266$ & 0.62 & $\mathrm{p}=0.11$ & $\mathbf{y}=0.0004 x+0.012$ & 0.36 & $\mathrm{p}=0.05$ \\
\hline
\end{tabular}

60

61

62

Table S6. Correlation of dicarboxylic acid with Fe

\section{Before Polar Sunrise After Polar Sunrise}

\begin{tabular}{lllllll} 
DCA vs Fe & Regression equation & $\mathbf{R}^{2}$ & P-value & Regression equation & $\mathbf{R}^{2}$ & P-value \\
\hline $\mathbf{C}_{\mathbf{2}}$ vs Fe & $\mathrm{y}=0.7418 \mathrm{x}-1.0494$ & 0.73 & $\mathrm{p}<0.05$ & $\mathrm{y}=0.4771 \mathrm{x}+3.0834$ & 0.26 & $\mathrm{p}>0.05$ \\
$\mathbf{C}_{\mathbf{3}}$ vs Fe & $\mathrm{y}=0.2658 \mathrm{x}-2.8929$ & 0.62 & $\mathrm{p}<0.05$ & $\mathrm{y}=0.1192 \mathrm{x}+1.9354$ & 0.45 & $\mathrm{p}>0.05$ \\
$\mathbf{C}_{\mathbf{4}}$ vs Fe & $\mathrm{y}=0.1999 \mathrm{x}-1.7053$ & 0.78 & $\mathrm{p}<0.05$ & $\mathrm{y}=0.0831 \mathrm{x}+4.3839$ & 0.13 & $\mathrm{p}>0.05$ \\
$\mathbf{C}_{\mathbf{5}}$ vs Fe & $\mathrm{y}=0.0865 \mathrm{x}-0.578$ & 0.80 & $\mathrm{p}<0.05$ & $\mathrm{y}=0.0729 \mathrm{x}+0.203$ & 0.34 & $\mathrm{p}>0.05$ \\
$\mathbf{C}_{\mathbf{6}}$ vs Fe & $\mathrm{y}=0.0691 \mathrm{x}-0.2824$ & 0.67 & $\mathrm{p}<0.05$ & $\mathrm{y}=0.0428 \mathrm{x}+0.208$ & 0.59 & $\mathrm{p}>0.01$ \\
$\mathbf{C}_{7}$ vs Fe & $\mathrm{y}=0.0069 \mathrm{x}-0.0227$ & 0.66 & $\mathrm{p}<0.05$ & $\mathrm{y}=0.007 \mathrm{x}-0.096$ & 0.71 & $\mathrm{p}>0.01$ \\
$\mathbf{C}_{\mathbf{8}}$ vs Fe & $\mathrm{y}=0.0039 \mathrm{x}+0.032$ & 0.54 & $\mathrm{p}>0.05$ & $\mathrm{y}=0.0041 \mathrm{x}-0.0129$ & 0.60 & $\mathrm{p}<0.05$ \\
$\mathbf{C}_{\mathbf{9}}$ vs Fe & $\mathrm{y}=0.0013 \mathrm{x}+0.1025$ & 0.08 & $\mathrm{p}>0.05$ & $\mathrm{y}=0.0015 \mathrm{x}+0.0536$ & 0.42 & $\mathrm{p}>0.05$
\end{tabular}

63

64

65

66

67

68 
69

Table S7. Statistics of Regression model: WSOC versus Fe, Cu and Mn (at Polar Sunrise)

71

72

73

74

75

76

77

78

79

80

81

82

83
Descriptive Statistics

\begin{tabular}{|l|r|r|r|}
\hline & \multicolumn{1}{|c|}{ Mean } & Std. Deviation & \multicolumn{1}{|c|}{ N } \\
\hline WSOC & 156.6436 & 90.25560 & 11 \\
Fe & 27.1691 & 16.40118 & 11 \\
Cu & .6282 & .26202 & 11 \\
Mn & .5900 & .38165 & 11 \\
\hline
\end{tabular}

Correlations

\begin{tabular}{|ll|r|r|r|r|}
\hline & & \multicolumn{1}{|c|}{ WSOC } & \multicolumn{1}{c|}{$\mathrm{Fe}$} & \multicolumn{1}{c|}{$\mathrm{Cu}$} & \multicolumn{1}{c|}{ Mn } \\
\hline Pearson Correlation & WSOC & 1.000 & .808 & .888 & .925 \\
& $\mathrm{Fe}$ & .808 & 1.000 & .731 & .899 \\
& $\mathrm{Cu}$ & .888 & .731 & 1.000 & .922 \\
& $\mathrm{Mn}$ & .925 & .899 & .922 & 1.000 \\
\hline Sig. (1-tailed) & WSOC &. & .001 & .000 & .000 \\
& $\mathrm{Fe}$ & .001 &. & .005 & .000 \\
& $\mathrm{Cu}$ & .000 & .005 &. & .000 \\
& $\mathrm{Mn}$ & .000 & .000 & .000 & \\
\hline N & $\mathrm{WSOC}$ & 11 & 11 & 11 & 11 \\
& $\mathrm{Fe}$ & 11 & 11 & 11 & 11 \\
& $\mathrm{Cu}$ & 11 & 11 & 11 & 11 \\
& $\mathrm{Mn}$ & 11 & 11 & 11 & 11 \\
\hline
\end{tabular}

Variables Entered/Removed ${ }^{a}$

\begin{tabular}{|l|l|l|l|}
\hline Model & \multicolumn{1}{|c|}{$\begin{array}{c}\text { Variables } \\
\text { Entered }\end{array}$} & \multicolumn{1}{|l|}{$\begin{array}{l}\text { Variables } \\
\text { Removed }\end{array}$} & \multicolumn{1}{|c|}{ Method } \\
\hline 1 & Mn & & $\begin{array}{l}\text { Stepwise } \\
\text { (Criteria: }\end{array}$ \\
& & & $\begin{array}{l}\text { Probability-of- } \\
\text { F-to-enter }<=. \\
\end{array}$ \\
& & & 050, \\
& & Probability-of- \\
& & & F-to-remove \\
& & & $>=.100$ ). \\
\hline
\end{tabular}

a. Dependent Variable: WSOC 


\begin{tabular}{|c|c|c|c|c|c|c|c|c|c|c|}
\hline & & & & & lodel Summar & & & & & \\
\hline \multirow[b]{2}{*}{ Model } & \multirow[b]{2}{*}{$\mathrm{R}$} & \multirow[b]{2}{*}{ R Square } & \multirow[b]{2}{*}{$\begin{array}{c}\text { Adjusted R } \\
\text { Square }\end{array}$} & \multirow[b]{2}{*}{$\begin{array}{l}\text { Std. Error of } \\
\text { the Estimate }\end{array}$} & \multicolumn{5}{|c|}{ Change Statistics } & \multirow[b]{2}{*}{$\begin{array}{l}\text { Durbin- } \\
\text { Watson }\end{array}$} \\
\hline & & & & & $\begin{array}{l}\text { R Square } \\
\text { Change }\end{array}$ & F Change & df1 & $\mathrm{df} 2$ & Sig. F Change & \\
\hline 1 & $.925^{\mathrm{a}}$ & .855 & .839 & 36.23009 & .855 & 53.060 & 1 & 9 & .000 & 1.325 \\
\hline
\end{tabular}

a. Predictors: (Constant), Mn

84

b. Dependent Variable: WSOC

ANOVA ${ }^{\text {a }}$

\begin{tabular}{|ll|l|r|r|r|r|}
\hline \multicolumn{2}{|c|}{} & \multicolumn{1}{|c|}{$\begin{array}{l}\text { Sum of } \\
\text { Squares }\end{array}$} & df & Mean Square & F & Sig. \\
\hline 1 & Regression & 69647.156 & 1 & 69647.156 & 53.060 & $.000^{\mathrm{b}}$ \\
& Residual & 11813.575 & 9 & 1312.619 & & \\
& Total & 81460.730 & 10 & & & \\
\hline
\end{tabular}

a. Dependent Variable: WSOC

85

b. Predictors: (Constant), Mn

\begin{tabular}{|c|c|c|c|c|c|c|c|c|c|c|c|c|c|}
\hline \multicolumn{14}{|c|}{ Coefficients ${ }^{a}$} \\
\hline \multirow[b]{2}{*}{ Model } & & \multicolumn{2}{|c|}{ Unstandardized Coefficients } & \multirow{2}{*}{$\begin{array}{c}\begin{array}{c}\text { Standardized } \\
\text { Coefficients }\end{array} \\
\text { Beta }\end{array}$} & \multirow[b]{2}{*}{$t$} & \multirow[b]{2}{*}{ Sig. } & \multicolumn{2}{|c|}{$95.0 \%$ Confidence Interval for B } & \multicolumn{3}{|c|}{ Correlations } & \multicolumn{2}{|c|}{ Collinearity Statistics } \\
\hline & & $B$ & Std. Error & & & & Lower Bound & Upper Bound & Zero-order & Partial & Part & Tolerance & VIF \\
\hline 1 & (Constant) & 27.631 & 20.809 & & 1.328 & .217 & -19.443 & 74.704 & & & & & \\
\hline & $\mathrm{Mn}$ & 218.666 & 30.019 & .925 & 7.284 & .000 & 150.758 & 286.574 & .925 & .925 & .925 & 1.000 & 1.000 \\
\hline
\end{tabular}

a. Dependent Variable:WSOC

Excluded Variables $^{a}$

\begin{tabular}{|c|c|c|c|c|c|c|c|c|}
\hline \multirow{2}{*}{\multicolumn{2}{|c|}{ Model }} & \multirow[b]{2}{*}{ Beta In } & \multirow[b]{2}{*}{$t$} & \multirow[b]{2}{*}{ Sig. } & \multirow[b]{2}{*}{$\begin{array}{c}\text { Partial } \\
\text { Correlation }\end{array}$} & \multicolumn{3}{|c|}{ Collinearity Statistics } \\
\hline & & & & & & Tolerance & VIF & $\begin{array}{l}\text { Minimum } \\
\text { Tolerance }\end{array}$ \\
\hline \multirow[t]{2}{*}{1} & $\mathrm{Fe}$ & $-.118^{b}$ & -.388 & .708 & -.136 & .192 & 5.207 & .192 \\
\hline & $\mathrm{Cu}$ & $.238^{b}$ & .703 & .502 & .241 & .149 & 6.710 & .149 \\
\hline
\end{tabular}

a. Dependent Variable: WSOC

b. Predictors in the Model: (Constant), Mn

86

87

88

89
Coefficient Correlations $^{a}$

\begin{tabular}{|c|c|c|c|}
\hline \multicolumn{3}{|c|}{ Model } & $\mathrm{Mn}$ \\
\hline \multirow[t]{2}{*}{1} & Correlations & $\mathrm{Mn}$ & 1.000 \\
\hline & Covariances & $\mathrm{Mn}$ & 901.153 \\
\hline
\end{tabular}

a. Dependent Variable: WSOC

Collinearity Diagnostics $^{a}$

\begin{tabular}{|c|c|c|c|c|c|}
\hline \multirow[b]{2}{*}{ Model } & \multirow[b]{2}{*}{ Dimension } & \multirow[b]{2}{*}{ Eigenvalue } & \multirow{2}{*}{$\begin{array}{c}\text { Condition } \\
\text { Index }\end{array}$} & \multicolumn{2}{|c|}{ Variance Proportions } \\
\hline & & & & (Constant) & $\mathrm{Mn}$ \\
\hline 1 & 1 & 1.851 & 1.000 & .07 & .07 \\
\hline & 2 & .149 & 3.526 & .93 & .93 \\
\hline
\end{tabular}

a. Dependent Variable: WSOC 
Residuals Statistics $^{\text {a }}$

\begin{tabular}{|l|r|r|r|r|r|}
\hline & Minimum & Maximum & \multicolumn{1}{c|}{ Mean } & Std. Deviation & \multicolumn{1}{|c|}{ N } \\
\hline Predicted Value & 66.9905 & 322.8299 & 156.6436 & 83.45487 & 11 \\
Std. Predicted Value & -1.074 & 1.991 & .000 & 1.000 & 11 \\
Standard Error of & 11.253 & 25.295 & 14.998 & 3.884 & 11 \\
Predicted Value & & & & & \\
Adjusted Predicted Value & 71.7158 & 363.1628 & 160.6045 & 90.66457 & 11 \\
Residual & -42.40990 & 76.58643 & .00000 & 34.37088 & 11 \\
Std. Residual & -1.171 & 2.114 & .000 & .949 & 11 \\
Stud. Residual & -1.635 & 2.229 & -.046 & 1.063 & 11 \\
Deleted Residual & -82.74279 & 85.17128 & -3.96089 & 44.24101 & 11 \\
Stud. Deleted Residual & -1.839 & 3.141 & .023 & 1.295 & 11 \\
Mahal. Distance & .056 & 3.965 & .909 & 1.085 & 11 \\
Cook's Distance & .002 & 1.271 & .169 & .374 & 11 \\
Centered Leverage Value & .006 & .397 & .091 & .109 & 11 \\
\hline
\end{tabular}

90

a. Dependent Variable: WSOC

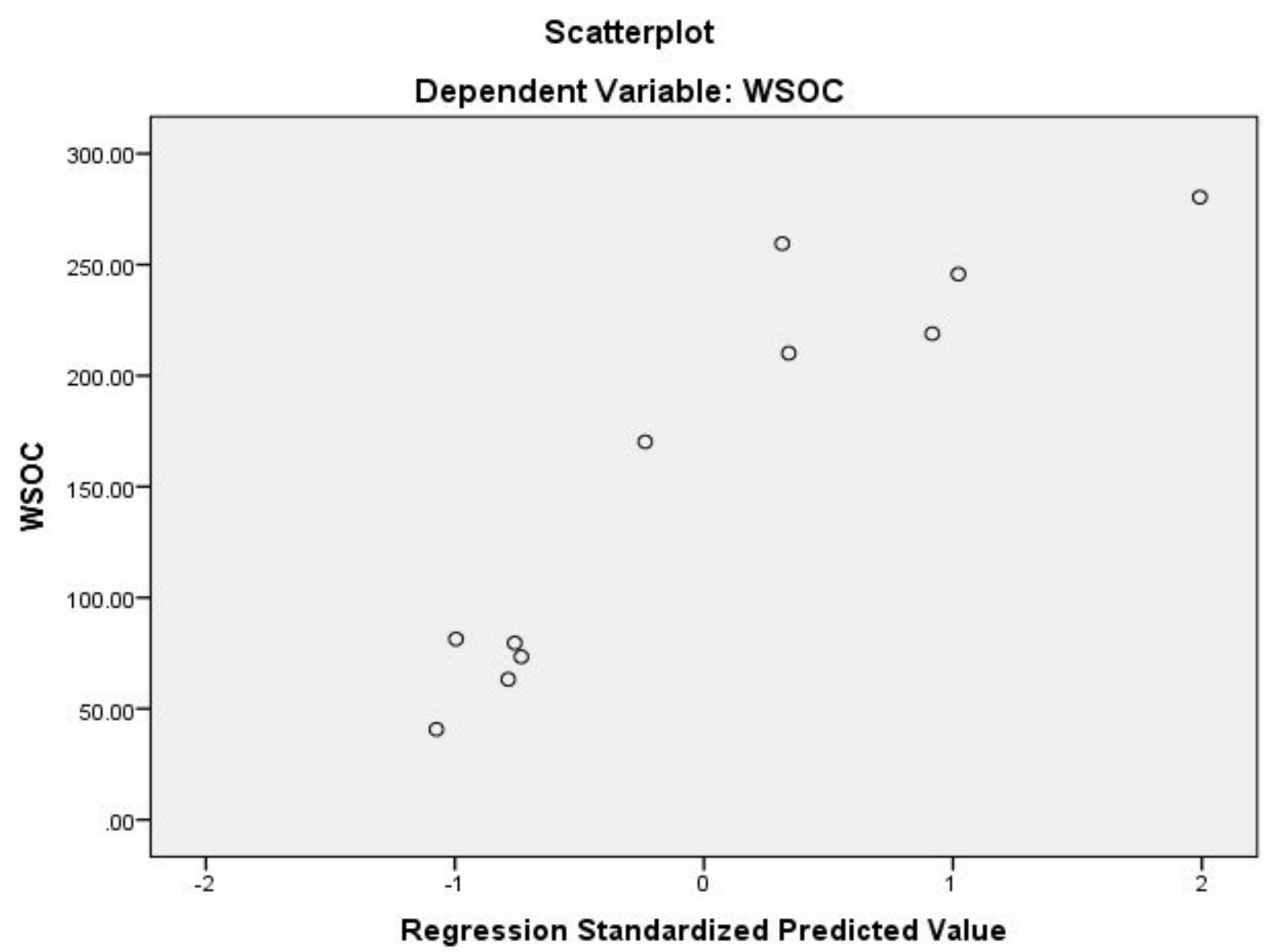

92 
WSOC Vs Fe, Cu and Mn (Multiple linear regression model) ANOVA $^{a}$

\begin{tabular}{|l|c|r|r|r|r|}
\hline Model & $\begin{array}{c}\text { Sum of } \\
\text { Squares }\end{array}$ & df & $\begin{array}{c}\text { Mean } \\
\text { Square }\end{array}$ & F & Sig. \\
\hline $1 \quad$ Regression & 70334.513 & 3 & 23444.838 & 14.750 & $.002^{\mathrm{b}}$ \\
& & & & & \\
$\quad$ Residual & 11126.217 & 7 & 1589.460 & & \\
$\quad$ Total & 81460.730 & 10 & & & \\
\hline
\end{tabular}

a. Dependent Variable: WSOC

b. Predictors: (Constant), Mn, Fe, Cu

Table S8. Analysis of variance (ANOVA) derived from the multiple linear regression model between one (WSOC) dependent and three $(\mathrm{Mn}, \mathrm{Fe}$ and $\mathrm{Cu}$ ) independent variables and ANOVA of each transition metals with WSOC. 


\section{Supporting figure}

Before Polar Sunrise

After Polar Sunrise
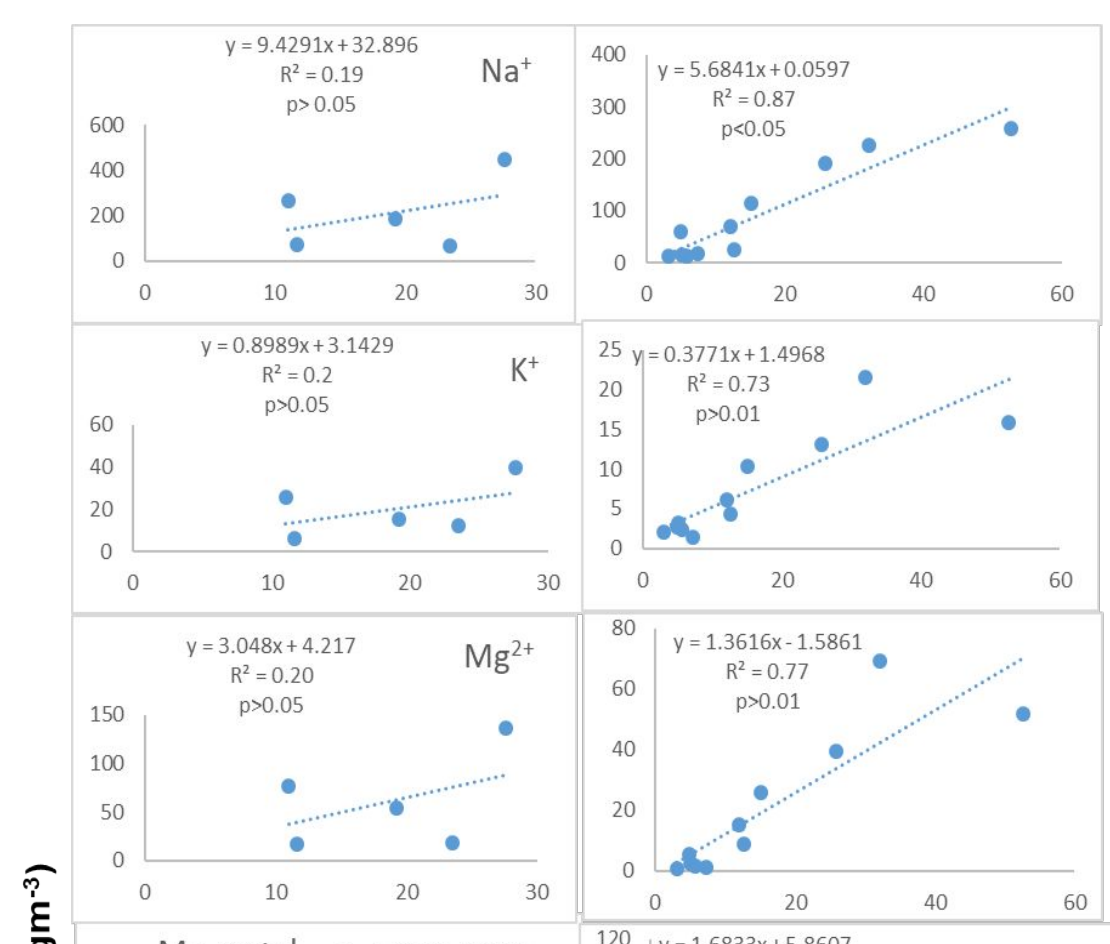

ڤ్
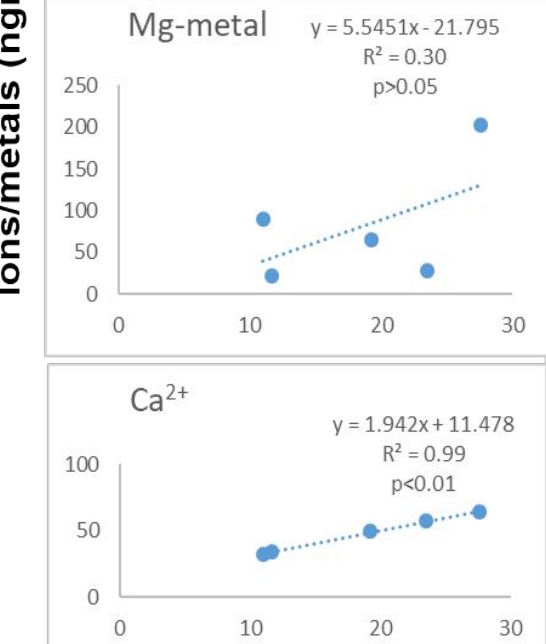

0
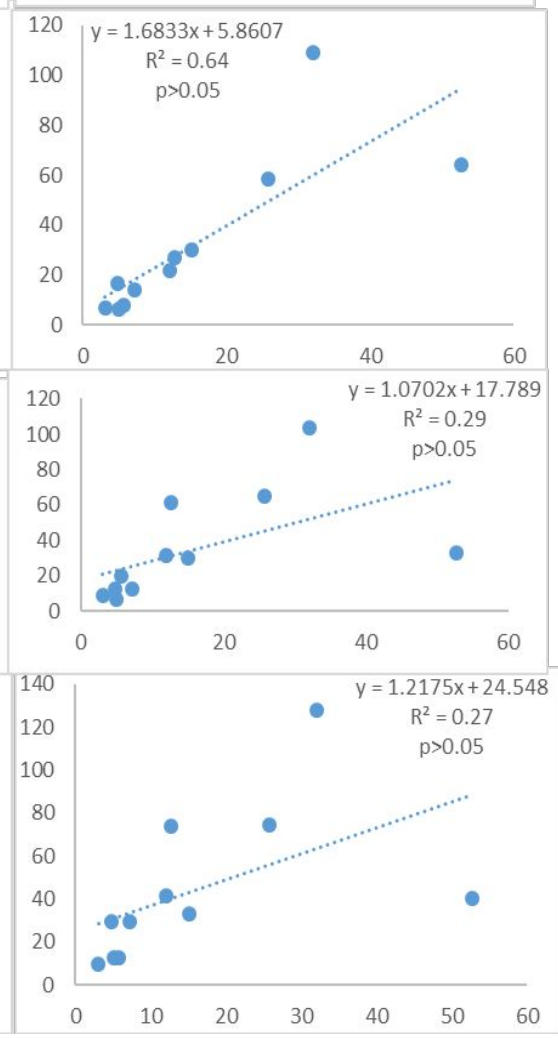

Oxalic acid $\left(\mathrm{C}_{2}\right) \mathrm{ngm}^{-3}$

Figure S1. Correlation of Oxalic acid with mono and divalent cations and alkaline earth metals 

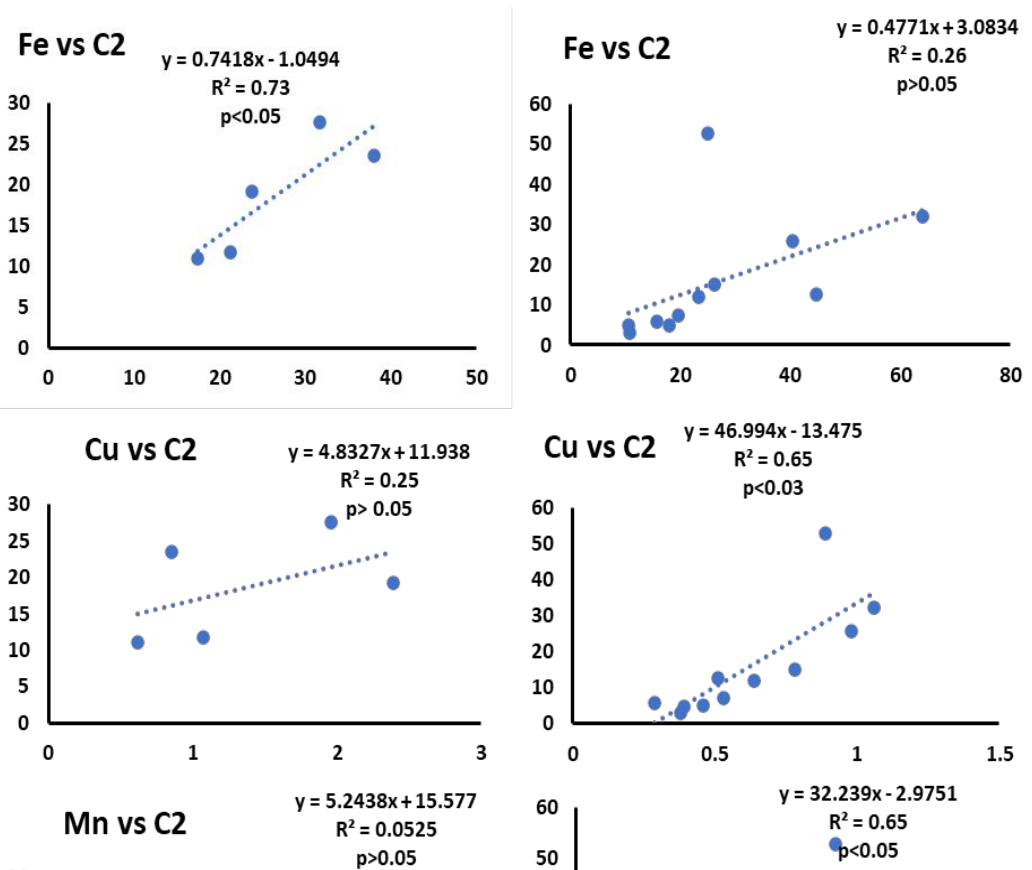

8
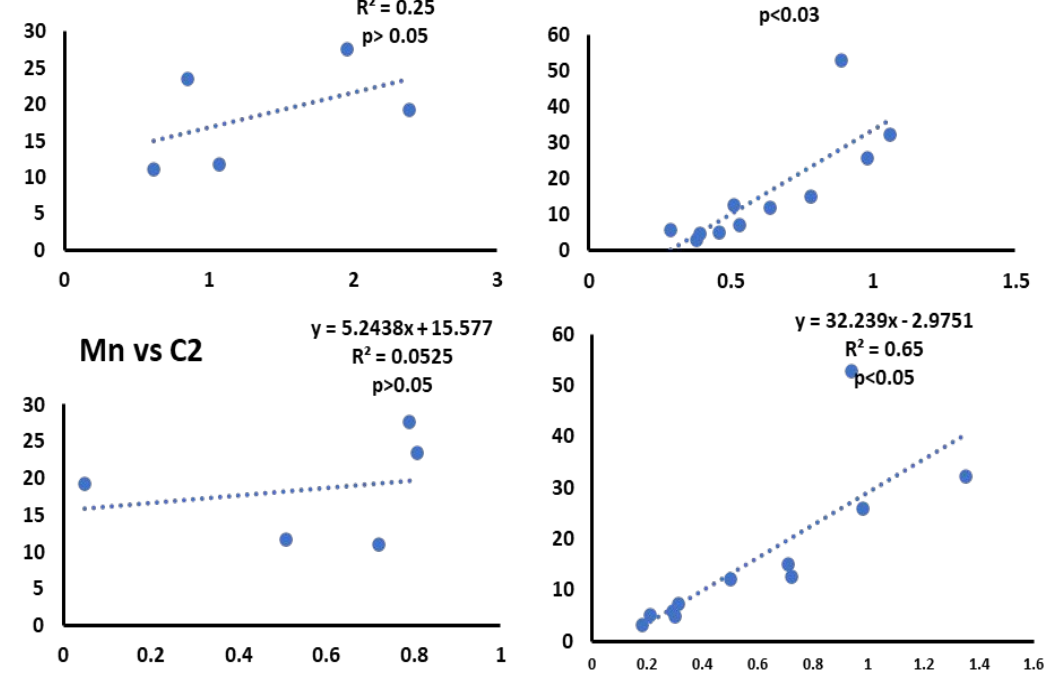

Transition metal $\left(\mathrm{ng} \mathrm{m}^{-3}\right)$

Figure S2. Correlation of oxalic acid with transition metals. 
198

199

200

201

202

203

204

205

206

207

208

209

210

211

212

213

214

215

216

217

218

219

220

221

222

\section{Residual Plots for WSOC}

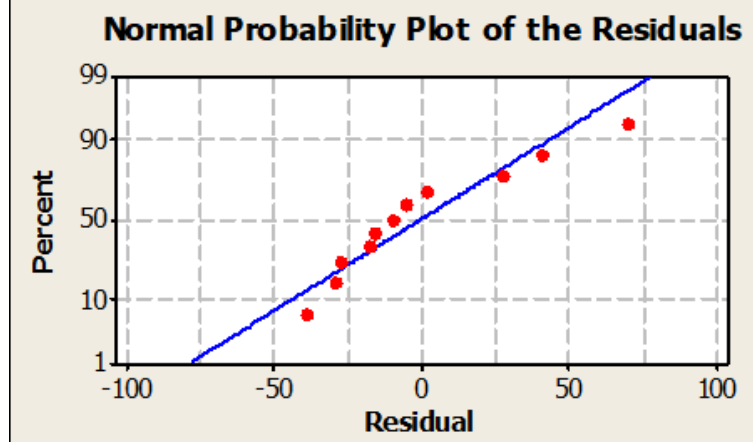

Residuals Versus the Fitted Values

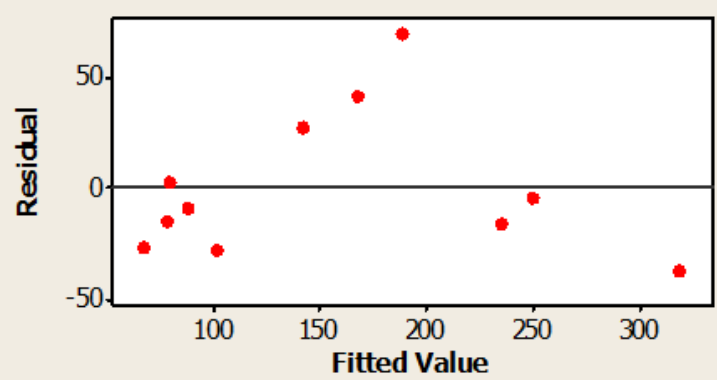

Histogram of the Residuals

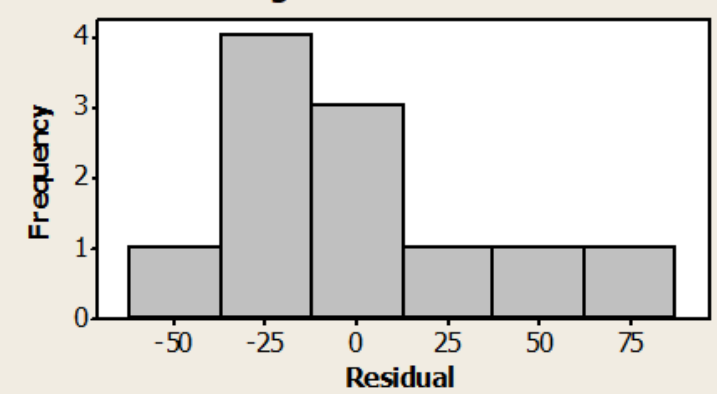

Residuals Versus the Order of the Data

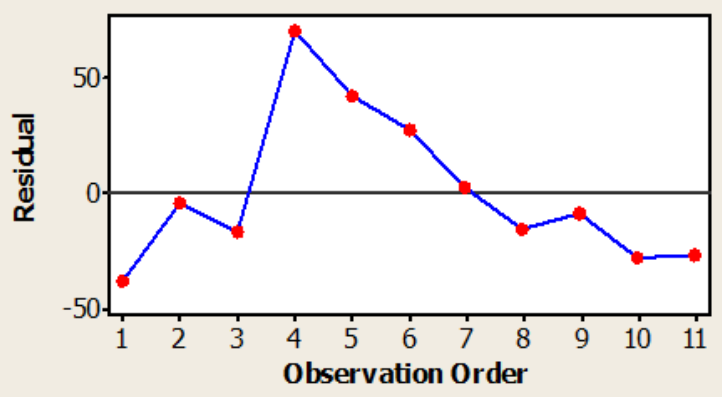

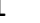

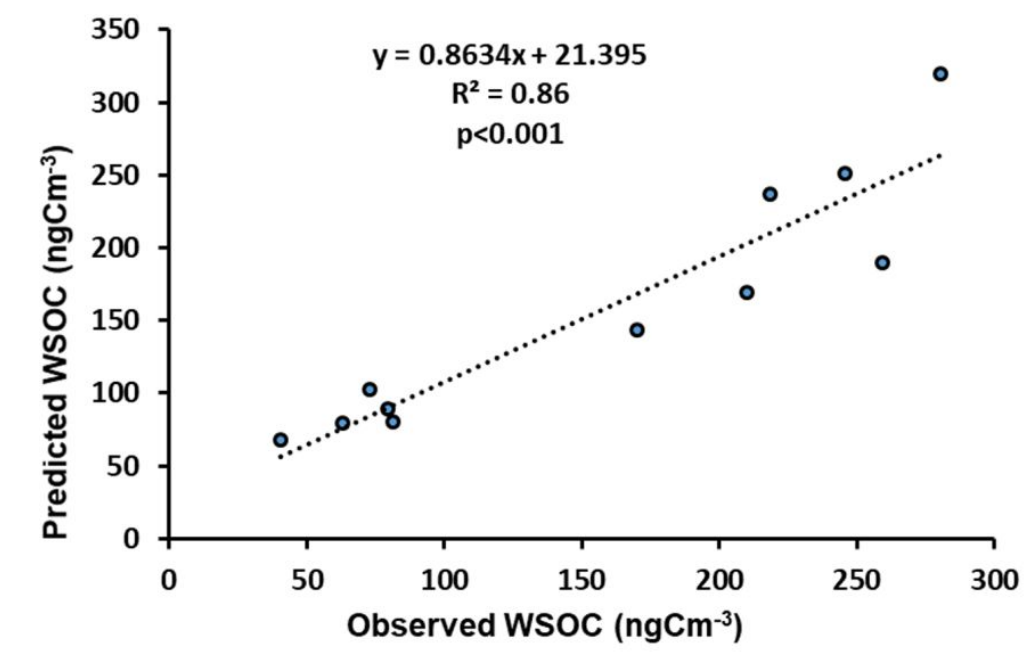

Figure S3. Residual plots for WSOC and correlation between predicted WSOC vs observed WSOC 


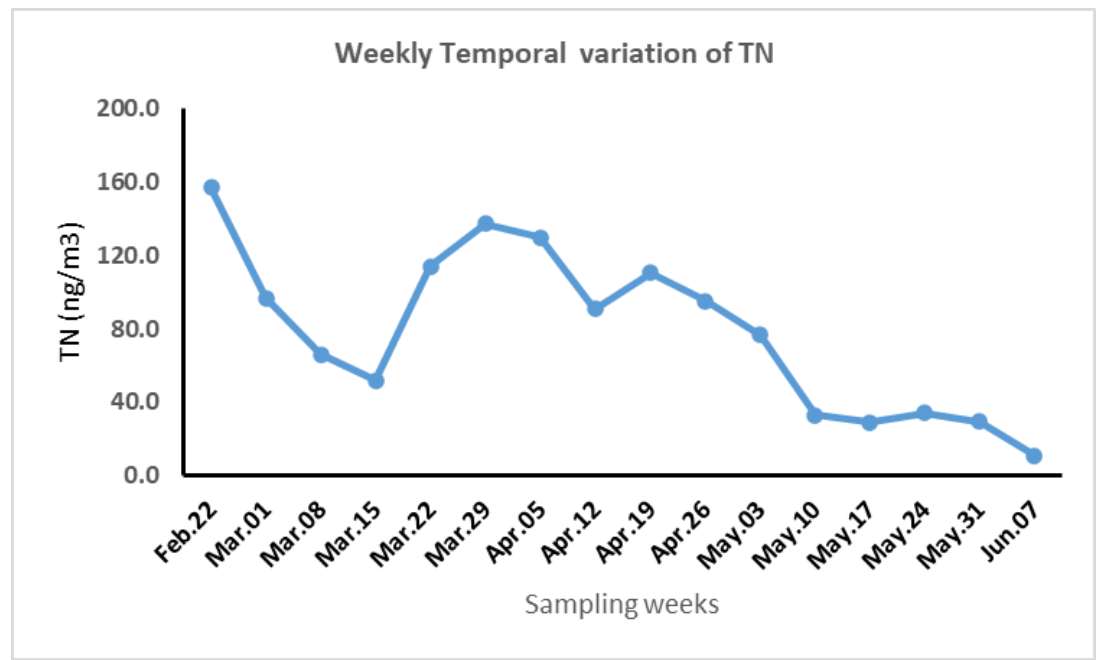

(a)

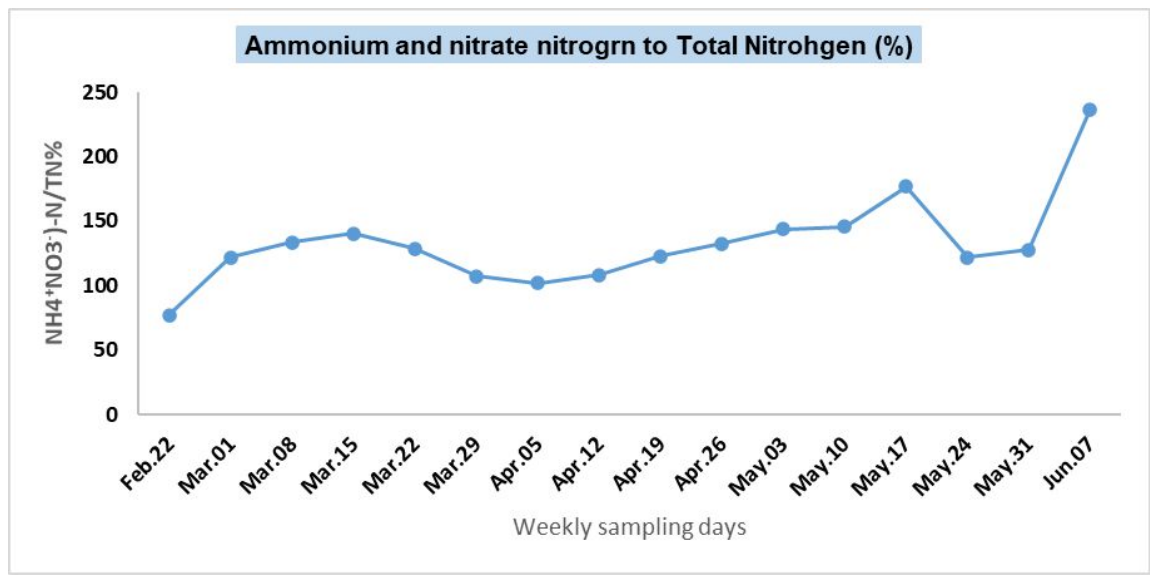

(b)

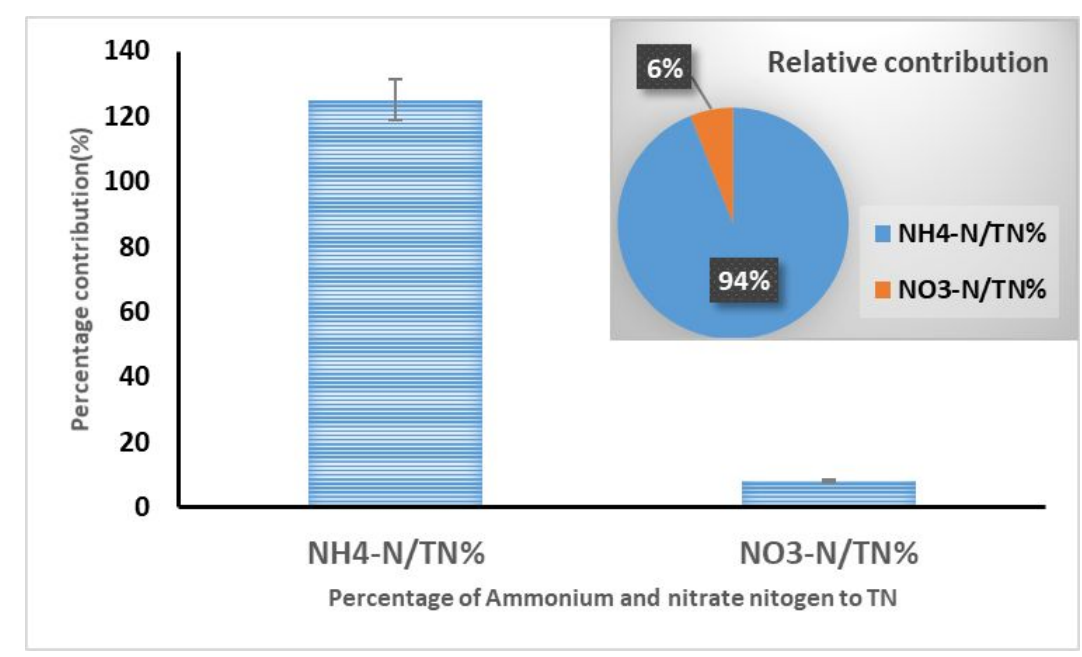

(c)

Figure S4. (a)Temporal weekly variations of Total nitrogen (TN) (b) contribution of ammonium and nitrate nitrogen to $\mathrm{TN}$ (c) relative contribution of ammonium and nitrate nitrogen to $\mathrm{TN}$ 

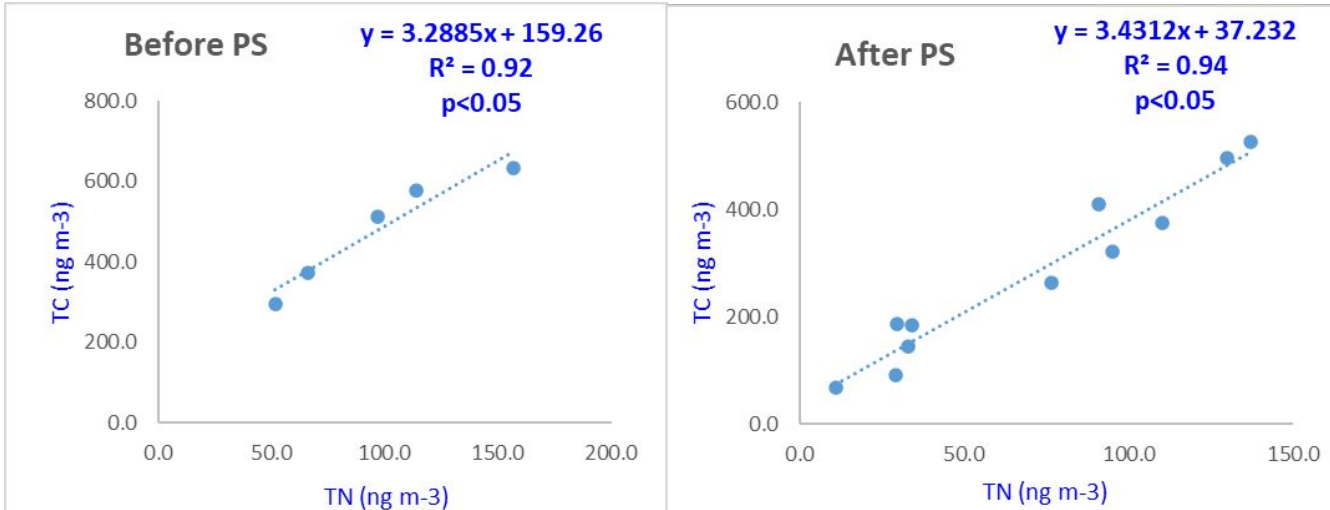

268

Figure S5. Correlation of total nitrogen (TN) with total carbon (TC) before and after polar 269 sunrise

270 